\title{
A generalization of Combinatorial Nullstellensatz
}

\author{
Michał Lasoń \\ Theoretical Computer Science Department, Faculty of Mathematics and Computer Science \\ Jagiellonian University, S. Łojasiewicza 6, 30-348 Kraków, Poland \\ Institute of Mathematics of the Polish Academy of Sciences \\ Św. Tomasza 30, 31-027 Kraków, Poland \\ mlason@tcs.uj.edu.pl
}

Submitted: Nov 9, 2009; Accepted: Oct 5, 2010; Published: Oct 15, 2010

Mathematics Subject Classification: 05E99, 05A99, 05C15

\begin{abstract}
In this note we give an extended version of Combinatorial Nullstellensatz, with weaker assumption on nonvanishing monomial. We also present an application of our result in a situation where the original theorem does not seem to work.
\end{abstract}

\section{Introduction}

The following theorem of Alon, known as Combinatorial Nullstellensatz, has numerous applications in Combinatorics, Graph Theory, and Additive Number Theory (see [1]).

Theorem 1. (Combinatorial Nullstellensatz [1]) Let $\mathbb{F}$ be an arbitrary field, and let $f$ be a polynomial in $\mathbb{F}\left[x_{1}, \ldots, x_{n}\right]$. Suppose the coefficient of $x^{\alpha_{1}} \cdots x_{n}^{\alpha_{n}}$ in $f$ is nonzero and $\operatorname{deg}(f)=\sum_{i=1}^{n} \alpha_{i}$. Then for any subsets $A_{1}, \ldots, A_{n}$ of $\mathbb{F}$ satisfying $\left|A_{i}\right| \geqslant \alpha_{i}+1$, there are $a_{1} \in A_{1}, \ldots, a_{n} \in A_{n}$ so that $f\left(a_{1}, \ldots, a_{n}\right) \neq 0$.

In this paper we extend this theorem by weakening the assumption on the degree of nonvanishing monomial. We also provide an explicit formula for coefficients of monomials in the usual expansion of $f$. Similar results were obtained independently by Schauz [5], however our proofs are simple and more direct. The paper is concluded with an application to a graph labeling problem for which classical approach does not seem to work.

\section{Generalized Combinatorial Nullstellensatz}

Let $\mathbb{F}$ be an arbitrary field, and let $f$ be a polynomial in $\mathbb{F}\left[x_{1}, \ldots, x_{n}\right]$. We define the support of $f$ by $\operatorname{Supp}(f):=\left\{\left(\alpha_{1}, \ldots, \alpha_{n}\right) \in \mathbb{N}^{n}\right.$ : the coefficient of $x_{1}^{\alpha_{1}} \cdots x_{n}^{\alpha_{n}}$ in $f$ is nonzero\}. On the set $\mathbb{N}^{n}$ and hence also on $\operatorname{Supp}(f)$ we have natural partial order: 
$\left(\alpha_{1}, \ldots, \alpha_{n}\right) \geqslant\left(\beta_{1}, \ldots, \beta_{n}\right)$ if and only if $\alpha_{i} \geqslant \beta_{i}$ for all $i$. The proof of the following theorem is a simple extension of an argument found by Michałek [4].

Theorem 2. (Generalized Combinatorial Nullstellensatz) Let $\mathbb{F}$ be an arbitrary field, and let $f$ be a polynomial in $\mathbb{F}\left[x_{1}, \ldots, x_{n}\right]$. Suppose that $\left(\alpha_{1}, \ldots, \alpha_{n}\right)$ is maximal in $\operatorname{Supp}(f)$. Then for any subsets $A_{1}, \ldots, A_{n}$ of $\mathbb{F}$ satisfying $\left|A_{i}\right| \geqslant \alpha_{i}+1$, there are $a_{1} \in A_{1}, \ldots, a_{n} \in$ $A_{n}$ so that $f\left(a_{1}, \ldots, a_{n}\right) \neq 0$.

Proof. The proof is by induction on $\alpha_{1}+\ldots+\alpha_{n}$. If $\alpha_{1}+\ldots+\alpha_{n}=0$, then $f \equiv c \neq 0$ and the assertion is true. If $\alpha_{1}+\ldots+\alpha_{n}>0$, then, without loss of generality, we can assume that $\alpha_{1}>0$. Fix $a \in A_{1}$ and divide $f$ by $\left(x_{1}-a\right)$. So, we have

$$
f=g \cdot\left(x_{1}-a\right)+h,
$$

where $\operatorname{deg}_{x_{1}}(h)=0$. This means that $h$ depends only on the variables $x_{2}, \ldots, x_{n}$. If there exists $a_{2} \in A_{2}, \ldots, a_{n} \in A_{n}$ so that $h\left(a_{2}, \ldots, a_{n}\right) \neq 0$, then we get $f\left(a, a_{2}, \ldots, a_{n}\right)=$ $h\left(a_{2}, \ldots, a_{n}\right) \neq 0$, which proves the assertion. Otherwise $\left.h\right|_{A_{2} \times \ldots \times A_{n}} \equiv 0$. By the division algorithm we have

$$
\operatorname{Supp}(g) \subseteq\left\{\left(\beta_{1}-r, \beta_{2}, \ldots, \beta_{n}\right):\left(\beta_{1}, \beta_{2}, \ldots, \beta_{n}\right) \in \operatorname{Supp}(f), 1 \leqslant r \leqslant \beta_{1}\right\},
$$

and $\left(\alpha_{1}-1, \alpha_{2}, \ldots, \alpha_{n}\right) \in \operatorname{Supp}(g)$. Thus the tuple $\left(\alpha_{1}-1, \alpha_{2}, \ldots, \alpha_{n}\right)$ is maximal in $\operatorname{Supp}(g)$. By inductive assumption we know that there exist $a_{1} \in A_{1} \backslash\{a\}, a_{2} \in$ $A_{2}, \ldots, a_{n} \in A_{n}$ so that $g\left(a_{1}, \ldots, a_{n}\right) \neq 0$. Hence

$$
f\left(a_{1}, a_{2}, \ldots, a_{n}\right)=\left(a_{1}-a\right) \cdot g\left(a_{1}, \ldots, a_{n}\right) \neq 0,
$$

which proves the assertion of the theorem.

\section{Coefficient formula}

Let $\mathbb{F}$ be an arbitrary field and let $A_{1}, \ldots, A_{n}$ be any finite subsets of $\mathbb{F}$. Define the function $N: A_{1} \times \ldots \times A_{n} \rightarrow \mathbb{F}$ by

$$
N\left(a_{1}, \ldots, a_{n}\right)=\prod_{i=1}^{n} \prod_{b \in A_{i} \backslash\left\{a_{i}\right\}}\left(a_{i}-b\right) .
$$

We may think of the function $N$ as a normalizing factor for the interpolating function on $A_{1} \times \ldots \times A_{n}$ defined by

$$
\chi_{\left(a_{1}, \ldots, a_{n}\right)}\left(x_{1}, \ldots, x_{n}\right)=N\left(a_{1}, \ldots, a_{n}\right)^{-1} \cdot \prod_{i=1}^{n} \prod_{b \in A_{i} \backslash\left\{a_{i}\right\}}\left(x_{i}-b\right) .
$$

Notice that $\chi_{\left(a_{1}, \ldots, a_{n}\right)}$ is everywhere zero on $A_{1} \times \ldots \times A_{n}$, except at the point $\left(a_{1}, \ldots, a_{n}\right)$ for which it takes the value of 1 .

We will need the following simple lemma. 
Lemma 1. Let $A$ be any finite subset of the field $\mathbb{F}$, with $|A| \geqslant 2$. Then

$$
\sum_{a \in A} \prod_{b \in A \backslash\{a\}}(b-a)^{-1}=0 .
$$

Proof. Consider the polynomial

$$
f(x)=\sum_{a \in A} \prod_{b \in A \backslash\{a\}} \frac{(x-b)}{(a-b)} .
$$

Its degree is at most $|A|-1$, and for all $a \in A$ it takes value of 1 . Hence $f \equiv 1$ and the coefficient of $x^{|A|-1}$ equals 0 . But it is also the same as the the left hand side of the asserted equality.

Theorem 3. (Coefficient Formula) Let $f$ be a polynomial in $\mathbb{F}\left[x_{1}, \ldots, x_{n}\right]$ and let $f_{\alpha_{1}, \ldots, \alpha_{n}}$ denote the coefficient of $x_{1}^{\alpha_{1}} \cdots x_{n}^{\alpha_{n}}$ in $f$. Suppose that there is no greater element than $\left(\alpha_{1}, \ldots, \alpha_{n}\right)$ in $\operatorname{Supp}(f)$. Then for any sets $A_{1}, \ldots, A_{n}$ in $\mathbb{F}$ such that $\left|A_{i}\right|=\alpha_{i}+1$ we have

$$
f_{\alpha_{1}, \ldots, \alpha_{n}}=\sum_{\left(a_{1}, \ldots, a_{n}\right) \in A_{1} \times \ldots \times A_{n}} \frac{f\left(a_{1}, \ldots, a_{n}\right)}{N\left(a_{1}, \ldots, a_{n}\right)} .
$$

Proof. The proof is by induction on the number of elements in the set

$$
\text { Cone }(f)=\left\{\beta \in \mathbb{N}^{n}: \text { there exists } \gamma \in \operatorname{Supp}(f) \text { and } \gamma \geqslant \beta\right\} \text {. }
$$

If $\mid$ Cone $(f) \mid=0$ then $f \equiv 0$ and the theorem is trivial. Otherwise let $\left(\beta_{1}, \ldots, \beta_{n}\right)$ be a maximal element of Cone $(f)$, so it also belongs to $\operatorname{Supp}(f)$. If $\left(\beta_{1}, \ldots, \beta_{n}\right)=\left(\alpha_{1}, \ldots, \alpha_{n}\right)$, then consider the polynomial

$$
f^{\prime}\left(x_{1}, \ldots, x_{n}\right)=f\left(x_{1}, \ldots, x_{n}\right)-f_{\alpha_{1}, \ldots, \alpha_{n}} \cdot \prod_{i=1}^{n} \prod_{b \in A_{i} \backslash\left\{a_{i}\right\}}\left(x_{i}-b\right)
$$

for arbitrary $a_{1} \in A_{1}, \ldots, a_{n} \in A_{n}$. Notice that

$$
\text { Cone }\left(f^{\prime}\right) \subset \text { Cone }(f) \backslash\left\{\left(\alpha_{1}, \ldots, \alpha_{n}\right)\right\} \text {, }
$$

so from inductive assumption we get the assertion for polynomial $f^{\prime}$. Since $\left(^{*}\right)$ is $\mathbb{F}$-linear and holds for $f^{\prime}$, it is enough to prove it for the polynomial

$$
h=f_{\alpha_{1}, \ldots, \alpha_{n}}^{-1} \cdot\left(f-f^{\prime}\right)=\prod_{i=1}^{n} \prod_{b \in A_{i} \backslash\left\{a_{i}\right\}}\left(x_{i}-b\right) .
$$

Now $h_{\alpha_{1}, \ldots, \alpha_{n}}=1$, and the right hand side of $(*)$ is also equal to 1 since $h\left(x_{1}, \ldots, x_{n}\right) \neq 0$ only for $\left(a_{1}, \ldots, a_{n}\right)$, so we are done.

If $\left(\beta_{1}, \ldots, \beta_{n}\right) \neq\left(\alpha_{1}, \ldots, \alpha_{n}\right)$ then we have $\left(\beta_{1}, \ldots, \beta_{n}\right) \ngtr\left(\alpha_{1}, \ldots, \alpha_{n}\right)$ by the assumptions. So there exists $i$ such that $\beta_{i}<\alpha_{i}$, without loss of generality we can assume that 
$\beta_{1}<\alpha_{1}$. Let $B_{1} \subset A_{1}$ be any subset with $\beta_{1}$ elements. So, we have $\left|A_{1} \backslash B_{1}\right| \geqslant 2$. Consider the polynomial

$$
f^{\prime}\left(x_{1}, \ldots, x_{n}\right)=f\left(x_{1}, \ldots, x_{n}\right)-f_{\beta_{1}, \ldots, \beta_{n}} \cdot x_{2}^{\beta_{2}} \cdots x_{n}^{\beta_{n}} \cdot \prod_{b \in B_{1}}\left(x_{1}-b\right) .
$$

As before we have that

$$
\operatorname{Cone}\left(f^{\prime}\right) \subset \operatorname{Cone}(f) \backslash\left\{\left(\beta_{1}, \ldots, \beta_{n}\right)\right\},
$$

so, from inductive assumption we get the assertion for polynomial $f^{\prime}$. It remains to prove it for the polynomial

$$
h=f_{\beta_{1}, \ldots, \beta_{n}}^{-1} \cdot\left(f-f^{\prime}\right)=x_{2}^{\beta_{2}} \cdots x_{n}^{\beta_{n}} \cdot \prod_{b \in B_{1}}\left(x_{1}-b\right) .
$$

Obviously, the left-hand side of equality $\left(^{*}\right)$ equals zero. After rewriting the right-hand side we get

$$
\begin{aligned}
& \sum_{\left(a_{1}, a_{2}, \ldots, a_{n}\right) \in A_{1} \times \ldots \times A_{n}}\left\{\prod_{i=1}^{n} \prod_{b \in A_{i} \backslash\left\{a_{i}\right\}}\left(b-a_{i}\right)\right\}^{-1} \cdot a_{2}^{\beta_{2}} \cdots a_{n}^{\beta_{n}} \cdot \prod_{b \in B_{1}}\left(a_{1}-b\right)= \\
= & \sum_{a_{2} \in A_{2}, \ldots, a_{n} \in A_{n}} \prod_{i=2}^{n} \prod_{b \in A_{i} \backslash\left\{a_{i}\right\}}\left(\left(b-a_{i}\right)^{-1} \cdot a_{i}^{\beta_{i}}\right) \cdot \\
& \cdot\left(\sum_{a_{1} \in A_{1}} \prod_{b \in A_{1} \backslash\left\{a_{1}\right\}}\left(b-a_{1}\right)^{-1} \prod_{b \in B_{1}}\left(a_{1}-b\right)\right)
\end{aligned}
$$

The last factor in this product can be simplified to the form

$$
\sum_{a_{1} \in A_{1} \backslash B_{1}} \prod_{b \in\left(A_{1} \backslash B_{1}\right) \backslash\left\{a_{1}\right\}}\left(b-a_{1}\right)^{-1},
$$

which is zero by the Lemma 1 . The proof is completed.

Notice that Theorem 3 implies Theorem 2. Indeed, if $f_{\alpha_{1}, \ldots, \alpha_{n}} \neq 0$, then $f$ cannot vanish on every point of $A_{1} \times \ldots \times A_{n}$. Also if $f_{\alpha_{1}, \ldots, \alpha_{n}}=0$, then either $f$ vanishes on the whole set $A_{1} \times \ldots \times A_{n}$, or there are at least two points for which $f$ takes a non-zero value.

\section{Applications}

In this section we give an example of possible application of Theorem 2. In some sense it generalizes the idea of lucky labelings of graphs from [2]. Given a simple graph $G=(V, E)$ and any function $c: V \rightarrow \mathbb{N}$, let $S(u)=\sum_{v \in N(u)} c(v)$ denote the sum of labels over the 
set $N(u)$ of all neighbors of $u$ in $G$. The function $c$ is called a lucky labeling of $G$ if $S(u) \neq S(w)$ for every pair of adjacent vertices $u$ and $w$. The main conjecture from [2] states that every $k$-colorable graph has a lucky labeling with values in the set $\{1,2, \ldots, k\}$. One of the results of [2] in this direction asserts that the set of labels $\{1,2,3\}$ is sufficient for every bipartite planar graph $G$. This result is a special case of the following general theorem.

Theorem 4. Let $G$ be a bipartite graph, which has an orientation with outgoing degree bounded by $k$. Suppose each vertex $v$ is equipped with a non-constant polynomial $f_{v} \in \mathbb{R}[x]$ of degree at most $l$ and positive leading coefficient. Then there is a labeling $c: V(G) \rightarrow$ $\{1,2, \ldots, k l+1\}$ such that for any two adjacent vertices $u$ and $w$,

$$
c(u)-\sum_{v \in N(u)} f_{v}(c(v)) \neq c(w)-\sum_{v \in N(w)} f_{v}(c(v)) .
$$

Proof. Assign to each vertex $v \in V(G)$ a variable $x_{v}$. Consider the polynomial

$$
h=\prod_{u w \in E(G)}\left(\sum_{v \in N(u)} f_{v}\left(x_{v}\right)+x_{w}-\sum_{v \in N(w)} f_{v}\left(x_{v}\right)-x_{u}\right)
$$

We want to show that we can choose values for $x_{v}$ from the set $\{1, \ldots, k l+1\}$ so that $h$ is non-zero. Let us fix an orientation of $G$ where outgoing degree is bounded by $k$. For each edge $u w \in E(G)$ oriented $u \rightarrow w$ choose the leading monomial in $f_{u}\left(x_{u}\right)$ from the factor corresponding to this edge in $h$. The product of these monomials over all edges of $G$ is a monomial $M$ of $h$ satisfying $\operatorname{deg}_{x_{v}}(M) \leqslant k l$ (since monomials from $f_{u}\left(x_{u}\right)$ are taken at most $k$ times). We claim that the coefficient of $M$ in $h$ is nonzero. Indeed, each time we take a product of monomials from factors of $h$ resulting in the monomial $M$, the sign of $M$ is the same (because $G$ is bipartite and leading coefficients of $f_{v}$ are positive). So the copies of $M$ cannot cancel as we are working in the field $\mathbb{R}$. Finally maximality of $M$ in $\operatorname{Supp}(h)$ can be seen easily by giving weight $1 / \operatorname{deg}\left(f_{u}\right)$ to variable $x_{u}, M$ is then of maximal degree. The assertion follows from Theorem 2.

Notice that in the above theorem the labels can be taken from arbitrary lists of size at least $k l+1$.

Let us conclude the paper with the following remark. Suppose that we want to use classical Combinatorial Nullstellensatz to the polynomial $f\left(x_{1}, \ldots, x_{n}\right)$ of degree $\sum_{i=1}^{n} \alpha_{i}$ with nonzero coefficient of $x_{1}^{\alpha_{1}} \cdots x_{n}^{\alpha_{n}}$. If $f\left(x_{1}, \ldots, x_{n}\right)=g\left(h\left(x_{1}\right), x_{2}, \ldots, x_{n}\right)$ with $\operatorname{deg}(h)=k$, then for arbitrary sets $A_{1}, A_{2}, \ldots, A_{n} \subset \mathbb{F}$, with $h(a) \neq h(b)$ for all distinct $a, b \in A_{1}$ and of size at least $\alpha_{1} / k+1, \alpha_{2}+1, \ldots, \alpha_{n}+1, f$ does not vanish on $A_{1} \times \ldots \times A_{n}$. So we gain almost $k$ times smaller first set in comparison with the classical version. It is an immediate consequence of the substitution $x_{1}^{\prime}:=h\left(x_{1}\right)$ and Theorem 2 applied to $f^{\prime}\left(x_{1}^{\prime}, x_{2}, \ldots, x_{n}\right)=f\left(x_{1}, \ldots, x_{n}\right)$. An analogous corollary is true for more variables being in fact equal to some polynomials.

Acknowledgement. I would like to thank Jarek Grytczuk for stimulating discussions on the polynomial method in Combinatorics. 


\section{References}

[1] N. Alon, Combinatorial Nullstellensatz, Comb. Prob. Comput. 8 (1999), 7-29.

[2] S. Czerwiński, J. Grytczuk, W. Żelazny, Lucky labelings of graphs, Inform. Process. Lett. 109 (2009), 1078-1081.

[3] O. Kouba, A duality based proof of the Combinatorial Nullstellensatz, Electron. J. Combin. 16 (2009), Note 9, 3 pp.

[4] M. Michałek, A short proof of Combinatorial Nullstellensatz, Amer. Math. Monthly (to appear).

[5] U. Schauz, Algebraically solvable problems: describing polynomials as equivalent to explicit solutions. Electron. J. Combin. 15 (2008), no. 1, Research Paper 10, 35 pp. 